Draft Version OCtober 1, 2018

Preprint typeset using $\mathrm{L}_{\mathrm{A}} \mathrm{T}_{\mathrm{E}} \mathrm{X}$ style emulateapj v. 5/2/11

\title{
THE SIZE OF THE NARROW-LINE EMITTING REGION IN THE SEYFERT 1 GALAXY NGC 5548 FROM EMISSION-LINE VARIABILITY
}

\author{
B. M. Peterson ${ }^{1,2}$, K. D. Denney ${ }^{1,3,4,5}$, G. De Rosa ${ }^{1,2}$, C. J. Grier ${ }^{1}$, \\ R. W. Pogge ${ }^{1,2}$, M. C. Bentz ${ }^{6}$, C. S. Kochanek ${ }^{1,2}$, M. Vestergand ${ }^{3,7}$, \\ E. Kilerci-Eser ${ }^{3}$, E. Dalla Bontà $\grave{n}^{8,9}$, And S. Ciroi ${ }^{8}$ \\ Draft version October 1, 2018
}

\begin{abstract}
The narrow [O III] $\lambda \lambda 4959,5007$ emission-line fluxes in the spectrum of the well-studied Seyfert 1 galaxy NGC 5548 are shown to vary with time. From this we show that the narrow line-emitting region has a radius of only $1-3 \mathrm{pc}$ and is denser $\left(n_{e} \sim 10^{5} \mathrm{~cm}^{-3}\right)$ than previously supposed. The [O III] line width is consistent with virial motions at this radius given previous determinations of the black hole mass. Since the [O III] emission-line flux is usually assumed to be constant and is therefore used to calibrate spectroscopic monitoring data, the variability has ramifications for the long-term secular variations of continuum and emission-line fluxes, though it has no effect on shorter-term reverberation studies. We present corrected optical continuum and broad $\mathrm{H} \beta$ emission-line light curves for the period 1988 to 2008.

Subject headings: galaxies: active — galaxies: individual (NGC 5548) - galaxies: nuclei — galaxies: Seyfert — quasars: emission lines
\end{abstract}

\section{INTRODUCTION}

Beyond the black-hole/accretion disk structure that forms the heart of an active galactic nucleus (AGN) and produces the thermal continuum spectrum, the standard paradigm to explain the ultraviolet through infrared spectra of AGNs features two distinct emission-line regions: a broad-line region (BLR), which consists of relatively dense clouds, filaments, or streams of gas deep in the gravitational potential well of the central black hole, and a lower-density, more spatially extended narrow-line region (NLR) that gives rise to the forbidden lines (e.g., [O III] $\lambda \lambda 4959,5007)$ and the narrow cores of the permitted lines. The size of the BLR, $R_{\mathrm{BLR}}$, is measured by reverberation mapping Blandford \& McKee 1982; Peterson 1993) and is found to be dependent on the luminosity of the AGN (Bentz et al. 2013, and references therein) roughly as expected from photoionization theory. Recent results using microlensing in gravitationally lensed quasars, while less developed, provide an independent confirmation of this picture (e.g., Guerras et al. 2013). In local Seyfert galaxies, the size of the BLR is typically light days to light weeks; even in the nearest AGNs, the BLR projects to only tens of microarcseconds, so it is currently resolvable only

\footnotetext{
${ }^{1}$ Department of Astronomy, The Ohio State University, 140 W 18th Ave, Columbus, OH 43210

${ }^{2}$ Center for Cosmology \& AstroParticle Physics, The Ohio State University, 191 West Woodruff Ave, Columbus, OH 43210

${ }^{3}$ Dark Cosmology Centre, Niels Bohr Institute, University of Copenhagen, Juliane Maries Vej 30, DK-2100 Copenhagen, Denmark

${ }^{4}$ Marie Curie Fellow

5 NSF Astronomy and Astrophysics Postdoctoral Fellow

${ }^{6}$ Department of Physics and Astronomy, Georgia State

University, 25 Park Place, Suite 610, Atlanta, GA 30303

7 Steward Observatory, University of Arizona, 933 North Cherry Avenue, Tucson, AZ 85721

8 Dipartimento di Fisica e Astronomia 'G. Galilei', Università di Padova, Vicolo dell'Osservatorio 3 I-35122, Padova, Italy

${ }^{9}$ INAF-Osservatorio Astronomico di Padova, Vicolo dell'Osservatorio 5 I-35122, Padova, Italy
}

by reverberation mapping or microlensing. The NLR, by contrast, is sometimes large enough to be partially resolved on the sky and often shows a clear biconical structure (e.g., Pogge 1988; Tadhunter \& Tsvetanov 1989; Schmitt, Storchi-Bergmann, \& Baldwin 1994; Wilson \& Tsvetanov 1994; Fischer et al. 2013).

The reverberation mapping technique relies on the intrinsic variability of the AGN continuum and the response of the broad emission lines to these variations, delayed on average by the light-travel time across the $\mathrm{BLR}, \tau_{\mathrm{BLR}}=R_{\mathrm{BLR}} / c$. The continuum and emission-line flux variations are not extremely large $(\sim 10-20 \%$ on broad-line reverberation timescales) so the reverberation technique requires quite precise spectrophotometric flux measurements, typically at the $1-2 \%$ level (Horne et al. 2004), to be successful. Unfortunately, even under excellent observing conditions, it is difficult to perform ground-based optical absolute spectrophotometry to better than $\sim 5 \%$ accuracy, so non-standard techniques must be employed to obtain higher internal precision. It is only necessary to have accurate relative flux calibration between epochs, and there are two standard strategies for doing so. The first strategy is to align the spectrograph slit so as to simultaneously record the spectra of the targeted AGN and a nearby non-variable star (Kaspi et al. 2000, and references therein). The second, more commonly used method is to assume that the flux in the forbidden [O III] $\lambda \lambda 4959,5007$ lines is constant on reverberation time scales and therefore can be used as an internal flux calibration standard (Foltz et al. 1981; Peterson et al. 1982).

The assumption that narrow-line fluxes are constant over reverberation timescales is justified by the spatial extent of the NLR. For case B recombination, the size of the NLR is of order

$$
R_{\mathrm{NLR}} \approx 19\left(\frac{L_{41}(\mathrm{H} \beta)}{\epsilon n_{3}^{2}}\right)^{1 / 3} \mathrm{pc}
$$


where $L_{41}(\mathrm{H} \beta)$ is the luminosity of the $\mathrm{H} \beta$ narrow component in units of $10^{41} \mathrm{ergs} \mathrm{s}^{-1}, n_{3}$ is the electron density in units of $10^{3} \mathrm{~cm}^{-3}$, and $\epsilon$ is the volume filling factor of the narrow-line gas (Peterson 1997). In addition to the large light-travel time across the NLR, the low particle density means that the recombination time is also long,

$$
\tau_{\text {rec }} \approx\left(n_{\mathrm{e}} \alpha_{\mathrm{B}}\right)^{-1} \approx 200 n_{3}^{-1} \text { years }
$$

where $\alpha_{\mathrm{B}}$ is the hydrogen case $\mathrm{B}$ recombination coefficient and has a value of $1.43 \times 10^{-13} \mathrm{~cm}^{3} \mathrm{~s}^{-1}$ at $T \approx 20,000 \mathrm{~K}$ (Osterbrock \& Ferland 2006). This effectively damps out any short timescale flux variations. The luminosity of the $\mathrm{H} \beta$ narrow component in NGC $5548(z=0.01718$, heliocentric $)$ is $\sim 4.3 \times 10^{40} \mathrm{ergs}$ $\mathrm{s}^{-1}$ (including a correction for Galactic absorption corresponding to $A_{B}=0.088 \mathrm{mag}$ ), and NLR electron densities are typically $n_{\mathrm{e}} \approx 2000 \mathrm{~cm}^{-3}($ Koski 1978), so for NGC 5548 , we expect $\tau_{\mathrm{NLR}}=R_{\mathrm{NLR}} / c>30$ years and $\tau_{\text {rec }} \approx 100$ years.

There are, however, only a small number of AGNs that have been spectroscopically monitored on timescales much longer than BLR reverberation timescales. In this regard, the Seyfert 1 galaxy NGC 5548 is a special case. It has been the subject of a number of spectroscopic monitoring programs, motivated at least in part by the desire to have a long time-series on at least one fairly typical intermediate luminosity AGN (e.g., Sergeev et al. 2007, and references therein); the optical spectroscopic coverage now extends over 40 years. A long baseline that includes multiple reverberation campaigns allows us, for example, to test the repeatability of black hole mass measurements as the BLR radius and emission-line profile can change on dynamical timescales $\left(\tau_{\mathrm{dyn}} \approx c \tau_{\mathrm{BLR}} / \Delta V\right.$, where $\Delta V$ is the broad line width) due to large-scale secular luminosity variations (e.g., Peterson et al. 2002; Gilbert \& Peterson 2003; Kilerci-Eser et al. 2013) that are potentially related to accretion-rate changes (e.g., MacLeod et al. 2010).

Absolute calibration of the thousands of optical spectra of NGC 5548 that have been obtained over recent decades has been tied to a fairly homogeneous subset of spectra that were obtained under photometric conditions during the first year of the 13-year International AGN Watch monitoring campaign on this source in 1988 89 (Peterson et al. 1991). Based on these observations, a flux of $F([\mathrm{O} \mathrm{III}] \lambda 5007)=5.58 \times 10^{-13} \mathrm{ergs} \mathrm{s}^{-1} \mathrm{~cm}^{-2}$ was adopted, and all subsequent spectra were scaled to this value. For the first seven years of this campaign, [O III $] \lambda 5007$ fluxes were measured in a similar high-quality subset of the spectra (Peterson et al. 1992, 1994, 1999; Korista et al. 1995) and were found to be consistent with this value to within the measurement errors, which are estimated to be $\sim 4.4 \%$ (see $₫ 2$ ), assuming that there is no real variability on time scales shorter than a few weeks.

In recent months, we have revisited the issue of absolute flux calibration of the NGC 5548 spectra motivated by identification of some apparent inconsistencies between recent and previously published measurements (Kilerci-Eser et al. 2013) and by the detection of strong long timescale narrow-line flux variability in another AGN in our monitoring program (De Rosa et al. 2013). Here we describe the results of this re-investigation, which reveals narrow-line flux variations on timescales short enough to allow determination of the size of the NLR. We discuss the data and measurements in $\S 2$. We discuss our recalibration of the historical light curve for NGC 5548 in $\S 3$ and the implications for the NLR in $\S 4$.

\section{DATA AND ANALYSIS}

We use measurements of the absolute flux of the [O III $] \lambda 5007$ emission line from high-quality CCD spectra that were obtained through wide entrance apertures to mitigate seeing effects (see Peterson et al. 1995) and under weather conditions that were recorded by the observer on site to be either "clear" or "photometric." In each case, the observing conditions are a judgment call, though the observers appeared to be quite conservative in declaring weather conditions to be sufficiently good that absolute spectrophotometry was possible. For the first 7 years of the International AGN Watch campaign (1988-89 to 1994-95), we used published [O III] $\lambda 5007$ emission-line fluxes (Peterson et al. 1992, 1994, 1999; Korista et al. 1995). New measurements were made from International AGN Watch data from Years 8 through 13 (Peterson et al. 2002). These measurements are given in Table 1.

In addition to the International AGN Watch data, we employed data from more recent monitoring campaigns that were undertaken by our group in cooperation with others. We refer to these data sets by the internal campaign names we used at MDM Observatory: AGN05 over 2005 March - April (Bentz et al. 2007), AGN07 over 2007 March - August (Dennev et al. 2010), and AGN12 over 2012 January - April (De Rosa et al. 2013). We also used data from a campaign undertaken by the Lick AGN Monitoring Program from 2008 February May (LAMP08; Bentz et al. 2009b). We again selected a subset of spectra for which the observer noted that the weather conditions were good. Measurements from all of these spectra are given in Table 2.

To estimate the uncertainty in each of the flux measurements in Tables 1 and 2, we assumed that the fractional uncertainty is constant for each set and that there is no true flux variability on the short timescales. We then compared flux differences between all pairs of measurements separated by no more than 10 to 20 days, depending on how many pairs were available, and assumed that variations on these timescales are due only to measurement errors. The fractional uncertainties we adopted are thus 0.044 for the International AGN Watch data, 0.040 for AGN05, 0.065 for AGN07, 0.055 for LAMP08, and 0.028 for AGN12.

As noted in $\$ 1$ there are good reasons to believe that the narrow-line flux should be non-variable on reverberation timescales. Moreover, we do not see any trend in the [O III] fluxes within the individual data sets that each span less than a year. We therefore average the flux measurements for each individual data set, and these values are given in Table 3 . We show these average [O III $] \lambda 5007$ fluxes as function of Julian Date in Figure 1 (bottom), along with the $5100 \AA$ continuum flux (top). There is a clearly significant long-term downward trend in the [O III $\lambda 5007$ flux as a function of time, that seems to bottom out and perhaps reverse in the more recent campaigns.

In order to measure directly the size of the [O III]- 
emitting region and to measure accurately the width of the [O III] lines in a high-resolution spectrum, we also retrieved images taken with the Hubble Space Telescope $(H S T)$ Space Telescope Imaging Spectrograph (STIS) from the Mikulsky Archive for Space Telescopes; Fischer et al. (2013) have previously characterized the [O III]-emitting region of NGC 5548 as "compact," based on these data.

\section{RECALIBRATION OF NGC 5548 LIGHT CURVES}

Figure 1 shows that our previous assumption that a single fixed value of the [O III $] \lambda 5007$ emission-line flux can serve as the absolute calibration for all NGC 5548 spectra over long timescales is incorrect. The [O III] $\lambda 5007$ emission-line flux varies significantly on timescales as short as a few years. It is therefore necessary to recalibrate the published $5100 \AA$ optical continuum and broad $\mathrm{H} \beta$ emission-line fluxes, correcting for the slow variations of the $[\mathrm{O}$ III $] \lambda 5007$ flux. The flux correction factors that need to be employed are given in Table 3 . We chose to use average values rather than to fit a smooth continuous function of time to all the data because the differences in the correction factors between closely spaced campaigns are very small and because we observed no trends within the individual campaigns.

If we want to place the entire $5100 \AA$ continuum light curve on a single flux scale, we must also take into account the different amounts of host-galaxy starlight contamination in each data set on account of the different spectrograph entrance apertures employed in the different observing campaigns. It should be noted that the International AGN Watch data sets have all been adjusted relative to a fixed entrance aperture of $5^{\prime \prime} .0 \times 7^{\prime \prime} .5$. In Table 4 , we give the host-galaxy fluxes through the various spectrograph entrance apertures employed in these campaigns based on modeling the HST Advanced Camera for Surveys (ACS) and Wide Field Camera 3 (WFC3) images of the host galaxy (Bentz et al. 2009a, 2013).

For each campaign, we multiply the published $5100 \AA$ optical continuum light curve by the appropriate correction factor in Table 3 and then subtract the appropriate host galaxy flux ${ }^{10}$ listed in Table 4 . The resulting hostcorrected light curve is shown in the top panel of Figure 2 and presented in Table 5 .

We also recalibrate the long-term emission-line light curve by multiplying the measurements from each data set by the flux correction factor from Table 3. We have previously determined from low-state spectra obtained in Years 2, 4, 9, 12 and 13 that the flux ratio of narrow $\mathrm{H} \beta$ to $[\mathrm{O}$ III $] \lambda 5007$ is constant and has a value of 0.11 (Peterson et al. 2004). This ratio is also the same in spectra from AGN05 and AGN07, so we conclude that narrow $\mathrm{H} \beta$ is also slowly varying. We therefore subtract off the narrow $\mathrm{H} \beta$ flux from the total $\mathrm{H} \beta$ flux, leaving behind the isolated broad component of $\mathrm{H} \beta$; this is the light curve shown in the lower panel of Figure 2 and presented in Table 6. We note in passing that the flux ratio [O III $] \lambda 5007 /[\mathrm{O}$ III $] \lambda 4959$ is fixed at 2.94 as both

10 The exception is the AGN07 data (Table 5 of Denney et al. 2010), which were already corrected for host-galaxy contribution using an earlier estimate from Bentz et al. (2009a). Here we add this earlier estimate back into the fluxes, apply the flux correction, and then subtract the new host galaxy correction. lines arise out of the same upper state $\left({ }^{1} D_{2}\right)$.

The recalibrated fluxes, particularly those obtained in low states, expose some of the limitations of the simple spectral analysis employed here. The simple prescription used for the $\mathrm{H} \beta$ flux measurement (Figure 1 of Peterson et al. 1991) was intended to capture most of the $\mathrm{H} \beta$ variations rather than accurate isolate the $\mathrm{H} \beta$ broad-line flux. For example, according to Table 6 , the broad $\mathrm{H} \beta$ flux reaches zero during the AGN07 campaign (at JD2454251), within the uncertainty of $\sim 4.7 \times 10^{-15} \mathrm{ergs} \mathrm{s}^{-1} \mathrm{~cm}^{-2}$. However, inspection of the original spectrum reveals a clear, but very weak broad component blended with other weak features. Measurement of very weak broad-line fluxes can be done accurately only by employing detailed decomposition and modeling of the individual spectra, which is work in progress.

\section{IMPLICATIONS FOR THE SIZE AND GAS DENSITY OF THE NARROW-LINE REGION}

As with the broad lines, the narrow-line light curve should be a shifted and smoothed version of the continuum driving the ionization. The temporal span of our light curves is too short to measure directly the lag between the continuum and the narrow-line fluxes. However, we can statistically estimate the timescale over which the continuum light curve must be smoothed in order to reproduce the narrow-line light curve. This timescale is roughly equal to the sum of the temporal smoothing created by the light travel time across the narrow line region and the recombination time scale from Equation 2,

$$
\tau_{\text {smooth }} \approx 2 R_{\text {NLR }} / c+\tau_{\text {rec }} \text {, }
$$

where we characterize the NLR as a sphere of radius $R_{\mathrm{NLR}}$.

We first need a statistical model for the continuum variability. We obtain this by fitting a damped random walk (DRW) stochastic process model to the $\mathrm{H} \beta$ light curve. The DRW model is known to well reproduce the variability of quasars (Kelly et al. 2009; Kozłowski et al. 2010; MacLeod et al. 2010, 2012; Zu et al. 2013). The optical continuum light curve in the most recent campaigns approaches zero flux in large part because of how the fluxes were measured, so we only fit the pre-2003 AGN Watch data. In any case, the large multi-year gaps in the later data would result in only weak constraints on the final model fit. We fit the light curve mean and the DRW time scale $\tau_{\text {damping }}$ and amplitude $\sigma$ using the procedures of Kozłowski et al. (2010) with Monte Carlo Markov Chains (MCMC) to estimate the uncertainties. Fits to the pseudo-magnitude $-2.5 \log F(\mathrm{H} \beta)$ (i.e., fractional variations in flux) had far higher maximum likelihoods than fits to the flux, so we only report those results, finding $\log \tau_{\text {damping }}=2.50, \log \sigma=-0.31$ and $\langle-2.5 \log F(\mathrm{H} \beta)\rangle=-2.00$ with $90 \%$ confidence ranges of $2.26<\log \tau_{\text {damping }}<3.04,-0.33<\log \sigma<-0.28$ and $-2.01<\langle-2.5 \log F(\mathrm{H} \beta)\rangle<-1.95$. Here $\tau_{\text {damping }}$ is in days and the fluxes in $\sigma$ and $F(\mathrm{H} \beta)$ were normalized by $10^{-15} \mathrm{ergs}^{-1} \mathrm{~cm}^{-2} \AA^{-1}$.

We drew model light curves from these MCMC chains, boxcar smoothed them on timescale $\tau_{\text {smooth }}$ and fit them to the seasonally averaged narrow-line light curve with a 
$\chi^{2}$ statistic. For the fit, we allowed a multiplicative scaling between the model of the smoothed continuum and the narrow-line data, needed for unit conversion, but no temporal shifts. We assigned the narrow-line data the epoch of the midpoint of the monitoring campaign. For each choice of $\tau_{\text {smooth }}$, we made $10^{5}$ trials and then constructed a Bayesian estimate of $P\left(\tau_{\text {smooth }}\right)$ with a logarithmic prior by weighting each trial as $\exp \left(-\chi^{2} / 2\right)$, summing over all the trials and normalizing the final distribution to unity. The resulting differential $P\left(\tau_{\text {smooth }}\right)$ and integral $P\left(>\tau_{\text {smooth }}\right)$ are shown in Figure 3. The median value is $\tau_{\text {smooth }}=14.8$ years and the $90 \%$ confidence range is 7.7 to 31.3 years. The narrow-line light curve is already smoothed over each season, with an average length of 0.72 years. We can roughly correct for this by adding the length of the average season to $\tau_{\text {smooth }}$, so we adopt as our final estimate $\tau_{\text {smooth }}=15.6$ years with a $90 \%$ confidence interval of 8.4 to 32.1 years.

Figure 4 shows the constraint this estimate of $\tau_{\text {smooth }}$ places on the size $R_{\mathrm{NLR}}$ of the emission region and the typical density $n_{e}$. For high densities, $\tau_{\text {smooth }}$ simply becomes the light-crossing time $2 R_{\mathrm{NLR}} / c$ of the NLR, and the density is required to be $n_{e} \gtrsim 10^{4} \mathrm{~cm}^{-3}$ to keep $\tau_{\text {rec }}<\tau_{\text {smooth. }}$. We also show the critical density for the $[\mathrm{O}$ III $] \lambda 5007$ transition $\left(n_{e}^{\text {crit }} \approx 7 \times 10^{5} \mathrm{~cm}^{-3}\right.$; Osterbrock \& Ferland 2006) and lines of constant $\mathrm{H} \beta$ luminosity $L(\mathrm{H} \beta)=4.3 \times 10^{40} \mathrm{ergs} \mathrm{s}^{-1}$ with a filling factor of $\epsilon=1$ and 0.01 , following Equation 1 . Peterson et al. (2004) estimate that the mass of the black hole in NGC 5548 is $M_{\mathrm{BH}}=7 \times 10^{7} M_{\odot}$. Ferrarese et al. (2001) measure the bulge velocity dispersion to be $\sigma_{*}=$ $183 \pm 10 \mathrm{~km} \mathrm{~s}^{-1}$ which together yield a black hole radius of influence $R_{\mathrm{BH}}=\left(G M_{\mathrm{BH}} / \sigma_{*}^{2}\right) \approx 9$ pc. Since this is larger than the estimated NLR radius, the [O III] line width of $460 \mathrm{~km} \mathrm{~s}^{-1}$ (see below) should be dominated by the gravity of the black hole, with $\Delta V^{2} \approx G M_{\mathrm{BH}} / R_{\mathrm{NLR}}$. This leads to a kinematic estimate of the size of the NLR $R_{\mathrm{NLR}} \approx 1.4 \mathrm{pc}$ which is in excellent agreement with all our other estimates, as shown in Figure 4, It is also noteworthy that this measurement is in excellent agreement with the size of the high-ionization component of the NLR predicted by photoionization equilibrium modeling (Kraemer et al. 1998).

The compactness of the [O III]-emitting NLR is surprising given earlier narrow-band imaging that indicated kpc-scale extended structure (Wilson et al. 1989). Our own Fabry-Perot [O III] $\lambda 5007$ images of NGC 5548 (Peterson et al. 1995) favor a compact NLR, but these images were obtained under poor seeing $\left(\sim 2^{\prime \prime}\right)$ conditions. We therefore inspected archival STIS images, as described in 42 . The [O III] lines extracted from these data are shown in Figure 5. The width of the lines is FWHM $=7.81 \AA=460 \mathrm{~km} \mathrm{~s}^{-1}$. In the spatial direction, we find that $94 \%$ of the [O III] emission is concentrated in a 2.42 pixel Gaussian core. A TinyTim (Krist, Hook, \& Stoehr 2011) model of the point-spread function for STIS imaging at the observed wavelength of [O III $] \lambda 5007$ has a width FWHM $=1.38$ pixels. Subtracting this in quadrature from the best-fit Gaussian to the [O III] emission gives the intrinsic width of the [O III]emitting region of 2 pixels or 0.102 arcsec or $37 \mathrm{pc}$. This means that $\sim 94 \%$ of the $[\mathrm{O}$ III] emission arises within a region of $R_{\mathrm{NLR}} \lesssim 18 \mathrm{pc}$, consistent with the results above.

\section{DISCUSSION AND CONCLUSIONS}

Flux variability and $H S T$ imaging both suggest that the [O III]-emitting region is much smaller than previously supposed, $1-3 \mathrm{pc}$ rather than kpc-scale. The actual physical extent of the NLR may be somewhat larger than the estimates given here if it is elongated along the line of sight and if, on account of obscuration, we preferentially detect emission from the narrow line-emitting gas on the near side. The [O III] $\lambda \lambda 4959,5007$ profiles shown in Figure 5 support such a scenario: the lines are distinctly asymmetric, with $\mathrm{HWHM}_{\text {blue }} / \mathrm{HWHM}_{\text {red }} \approx 2.12$. Such blueward asymmetries are often assumed to indicate that the NLR has an outflowing component (e.g., (e.g. Glaspev et al. 1976; Glaspey, Walker, \& Stockton 1976; Pelat \& Alloin 1980; Heckman et al. 1981; Peterson. Foltz. \& Bvard 1981; Véron 1981; Whittle 1985), which modern spatially resolved studies have confirmed (e.g., Storchi-Bergmann 2012). But the outflow velocities in any case are not terribly different from the virial velocity and none of our conclusions about the compactness of the NLR would be significantly altered if the NLR has an outflowing component.

In addition to being compact, we also infer that the particle density in the [O III]-emitting region is higher than previously supposed. This seems to be consistent with other observations: it is well known that the widths of the narrow lines in Seyfert 2 galaxies correlate with both critical density and ionization potential (Pelat. Alloin. \& Fosburv 1981; Filippenko \& Halpern 1984; DeRobertis \& Osterbrock 1984; Espey et al. 1994). Presumably, the higher velocity gas is closer to the central source, so we can explain this as a consequence of ionization stratification. From the [O III] flux variations, we infer that the electron density is $\sim 10^{5} \mathrm{~cm}^{-3}$, close to the critical density.

We suspect that this is also true for the NLR in other AGNs and there have simply not been enough high-quality spectrophotometric observations over a sufficiently long timescale for narrow-line variability to have been reliably detected in many cases. Indeed, we have found only one other credible report in the refereed literature of narrow emission-line flux variability, the case of 3C 390.3 (Zheng et al. 1995). This suggests that a systematic search of long-term changes in NLR fluxes would be rewarding and that, in principle, the time variability of the NLR would provide an independent check of black hole mass estimates from reverberation mapping of the BLR.

The narrow-line flux variations that we report on here do not negate any of the conclusions to date that have been drawn from reverberation mapping studies. The narrow-line fluxes are still effectively constant on timescales much longer than reverberation timescales. Indeed, if the narrow-line gas is virialized, as the broad-line gas seems to be, then $\tau_{\mathrm{NLR}} / \tau_{\mathrm{BLR}} \approx$ $(\Delta V$ [broad $] / \Delta V$ [narrow $])^{2}$, which is of order 100 . These results do, however, show that absolute calibration of the narrow-line fluxes requires attention.

We are grateful to the National Science Foundation 
for support of this work through grants AST-1008882 (BMP, GDR, CJG, and RWP) and AST-1009756 (CSK) to The Ohio State University, and PHY11-25915 (MV) to the Kavli Institute for Theoretical Physics, a CAREER Grant AST-1253702 (MCB) to Georgia State University, and a Postdoctoral Research Fellowship AST-1302093 (KDD). CJG is grateful for support through an Presidential Fellowship from The Ohio State University. EDB is supported by Padua University through grants 60A02$1283 / 10$, 60A02-5052/11, and 60A02-4807/12. MV thanks the Kavli Institute for Theoretical Physics at Uni- versity of California, Santa Barbara, for their hospitality where part of this work was done. The research leading to these results has received funding from the People Programme (Marie Curie Actions) of the European Union's Seventh Framework Programme FP7/2007-2013/ under REA grant agreement No. 300553 (KDD). The Dark Cosmology Centre is funded by the Danish National Research Foundation. This research has made use of the NASA/IPAC Extragalactic Database (NED), which is operated by the Jet Propulsion Laboratory, California Institute of Technology, under contract with the National Aeronautics and Space Administration.

\section{REFERENCES}

Bentz, M. C., et al. 2007, ApJ, 662, 205

Bentz, M. C., et al. 2009a, ApJ, 697, 160

Bentz, M. C., et al. 2009b, ApJ, 705, 199

Bentz, M. C., et al. 2013, ApJ, 767:149

Blandford, R. D., \& McKee, C. F. 1982, ApJ, 255, 419

Denney, K. D., et al. 2010, ApJ, 721, 715

De Robertis, M.M., \& Osterbrock, D.E. 1984, ApJ, 286, 171

De Rosa, G., et al., in preparation

Espey, B.R., et al. ApJ, 434, 484

Ferrarese, L., Pogge, R.W., Peterson, B.M., Merritt, D., Wandel, A., Joseph, C.L. 2001, ApJ, 555, L79

Filippenko, A.V., \& Halpern, J.P. 1984, ApJ, 285, 458

Fischer, T.C., Crenshaw, D.M., Kraemer, S.B., \& Schmitt. H.R. 2013, ApJS, in press (arXiv:1308.4129)

Foltz, C.B., Peterson, B.M., Capriotti, E.R., Byard, P.L. Bertram, R., \& Lawrie, D.G. 1981, ApJ, 250, 508

Gilbert, K.M., \& Peterson, B.M. 2003, ApJ, 587, 123

Glaspey, J.W., Eilek, J.A., Fahlman, G.G., \& Auman, J.R. 1976, ApJ, 203, 335

Glaspey, J.W., Walker, G.A.H., \& Stockton, A. 1976, ApJ, 210, 27

Guerras, E., Mediavilla, E., Jimenez-Vicente, J., Kochanek, C.S., Muñoz, J.A., Falco, E., \& Motta, V. 2013, ApJ, 764:160

Heckman, T.M., Miley, G.K., van Breugel, W.J.M., \& Butcher, H.R. 1981, ApJ, 247, 403

Horne, K., Peterson, B.M., Collier, S.J., \& Netzer, H. 2004, PASP, 116, 465

Kaspi, S., Smith, P.S., Netzer, H., Maoz, D., Jannuzi, B.T., \& Giveon, U. 2000, ApJ, 533, 631

Kelly, B.C., Bechtold, J., \& Siemiginowska, A. 2009, ApJ, 698, 895

Kilerci-Eser, E., Vestergaard, M., Peterson, B.M., Bentz, M.C., \& Denney, K.D. 2013, in preparation

Korista, K.T., et al. 1995, ApJS, 97, 285

Koski, A.T. 1978, ApJ, 223, 56

Kozłowski, S., Kochanek, C.S., Udalski, A., Wyrzykowski, Ł., Soszyński, I., Szymański, M.K., Kubiak, M., Pietrzyński, G., Szewczyk, O., Ulaczyk, K., \& Poleski, R. 2010, ApJ, 708, 927

Kraemer, S.B., Crenshaw, D.M., Filippenko, A.V., \& Peterson, B.M. 1998, ApJ, 499, 719

Krist, J.E., Hook, R.N., \& Stoehr, F. 2011, Proceedings of SPIE, Vol. $8127,8127 \mathrm{~J}-1$
MacLeod, C.L., Ivezić, Z̆., Kochanek, C.S. Kozłowski, S., Kelly, B., Bullock, E., Kimball, A., Sesar, B., Westman, D., Brooks, K. Gibson, R., Becker, A.C., \& de Vries, W.H. 2010, ApJ, 721, 1014

MacLeod, C.L., Ivezić, Z., Sesar, B., de Vries, W., Kochanek, C.S., Kelly, B.C., Becker, A.C., Lupton, R.H., Hall, P.B., Richards, G.T., Anderson, S.F., \& Schneider, D.P. 2012, ApJ, 753: 106

Osterbrock, D.E., \& Ferland, G.J. 2006, Astrophysics of Gaseous Nebulae and Active Galactic Nuclei, (Sausalito: University Science Books)

Pelat, D., \& Alloin, D. 1980, A\&A, 81, 172

Pelat, D., Alloin, D., \& Fosbury, R.A.E. 1981, MNRAS, 195, 787 Peterson, B.M., 1993, PASP, 105, 247

Peterson, B.M. 1997, An Introduction to Active Galactic Nuclei, (Cambridge: Cambridge Univ. Press), p. 101

Peterson, B.M., Foltz, C.B., \& Byard, P.L. 1981, ApJ, 243, L61

Peterson, B.M., Foltz, C.B., Byard, P.L., \& Wagner, R.M. 1982, ApJS, 49, 469

Peterson, B.M., Pogge, R.W., Wanders, I., Smith, S.E., \&

Romanishin, W. 1995, PASP, 107, 579

Peterson, B.M., et al. 1991, ApJ, 368, 119

Peterson, B.M., et al. 1992, ApJ, 392, 470

Peterson, B.M., et al. 1994, ApJ, 425, 622

Peterson, B.M., et al. 1999, ApJ, 510, 659

Peterson, B.M., et al. 2002, ApJ, 581, 197

Peterson, B.M., et al. 2004, ApJ, 613, 682

Pogge, R.W. 1988, ApJ, 328, 519

Schmitt, H.R., Storchi-Bergmann, T., \& Baldwin, J.A. 1994, ApJ, 423,237

Sergeev, S.G., Doroshenko, V.T., Dzyuba, S.A., Peterson, B.M., Pogge, R.W., \& Pronik, V.I. 2007, ApJ, 668, 708

Storchi-Bergmann, T. 2012, in AGN Winds in Charleston, ed. G. Chartas, F. Hamann, \& K.M. Leighly (San Francisco: Astronomical Society of the Pacific), p. 133

Tadhunter, C., \& Tsvetanov, Z. 1989, Nature, 341, 422

Véron, M.P. 1981, A\&A, 100, 12

Whittle, D.M. 1985, MNRAS, 213, 1

Wilson, A.S., \& Tsvetanov, Z. 1994, AJ, 107, 1227

Wilson, A.S., Wu, X., Heckman, T.M., Baldwin, J.A., \& Balick, B. 1989, ApJ, 339, 729

Zheng, W., Pérez, E., Grandi, S.A., \& Penston, M.V. 1995, AJ, 109, 2355

Zu, Y., Kochanek, C.S., Kozłowski, S., \& Udalski, A. 2013, ApJ, 765:196 
TABLE 1

Unpublished AGN Watch Measurements

\begin{tabular}{|c|c|c|}
\hline $\begin{array}{l}\text { UT Date } \\
(1)\end{array}$ & $\begin{array}{l}\text { File Name } \\
(2)\end{array}$ & $\begin{array}{c}F([\mathrm{O} \text { III }] \\
(3)\end{array}$ \\
\hline 1995 Nov 27 & n50052h & $5.21 \pm 0.23$ \\
\hline 1996 Jan 11 & n50093a & $5.03 \pm 0.22$ \\
\hline 1996 Apr 02 & n50175a & $5.30 \pm 0.23$ \\
\hline 1996 Jun 28 & n50262a & $4.93 \pm 0.22$ \\
\hline 1996 Sep 10 & n50336h & $4.76 \pm 0.21$ \\
\hline 1997 Feb 14 & n50493a & $5.14 \pm 0.23$ \\
\hline 1997 Jun 24 & n50623a & $4.88 \pm 0.22$ \\
\hline 1997 Jul 06 & n50635a & $5.10 \pm 0.22$ \\
\hline 1997 Aug 04 & n50664h & $5.20 \pm 0.23$ \\
\hline 1997 Sep 06 & n50697h & $5.49 \pm 0.24$ \\
\hline 1998 Jan 25 & n50838a & $5.16 \pm 0.23$ \\
\hline 1998 Mar 03 & n50875a & $4.94 \pm 0.22$ \\
\hline 1998 May 22 & n50955a & $5.02 \pm 0.22$ \\
\hline 1998 Jul 23 & n51017h & $5.00 \pm 0.22$ \\
\hline 1998 Aug 31 & n51056h & $5.52 \pm 0.24$ \\
\hline 1998 Sep 17 & n51077h & $4.44 \pm 0.20$ \\
\hline 1999 Jan 10 & n51189h & $4.67 \pm 0.20$ \\
\hline 1999 Feb 12 & n51221h & $4.66 \pm 0.20$ \\
\hline 1999 Feb 23 & n51233h & $5.27 \pm 0.23$ \\
\hline 1999 Mar 12 & n51250h & $5.08 \pm 0.22$ \\
\hline 1999 Apr 24 & n51293h & $5.06 \pm 0.22$ \\
\hline 1999 Jul 09 & n51368h & $5.10 \pm 0.22$ \\
\hline 1999 Jul 18 & n51377h & $5.11 \pm 0.22$ \\
\hline 1999 Aug 17 & n51407h & $5.44 \pm 0.23$ \\
\hline 1999 Sep 10 & n51431h & $4.81 \pm 0.21$ \\
\hline 2000 Dec 21 & n51900h & $4.82 \pm 0.21$ \\
\hline
\end{tabular}

Note. - Column (1) gives the UT Date of the observation. Column (2) gives the name of the spectrum as it appears in the International AGN Watch archive (see http://www.astronomy.ohio-state.edu/ agnwatch), where the prefix "n5" refers to the galaxy, the following digits are the four least-significant figures in the Julian Date of observation, and the final letter indicates the origin of the data (see Table 2 of Peterson et al. 2002). The measured observed frame [O III] $\lambda 5007$ flux appears in column (3), in units of $10^{-13} \mathrm{ergs} \mathrm{s}^{-1} \mathrm{~cm}^{-2}$. 
TABLE 2

Measurements from Recent Monitoring Campaigns

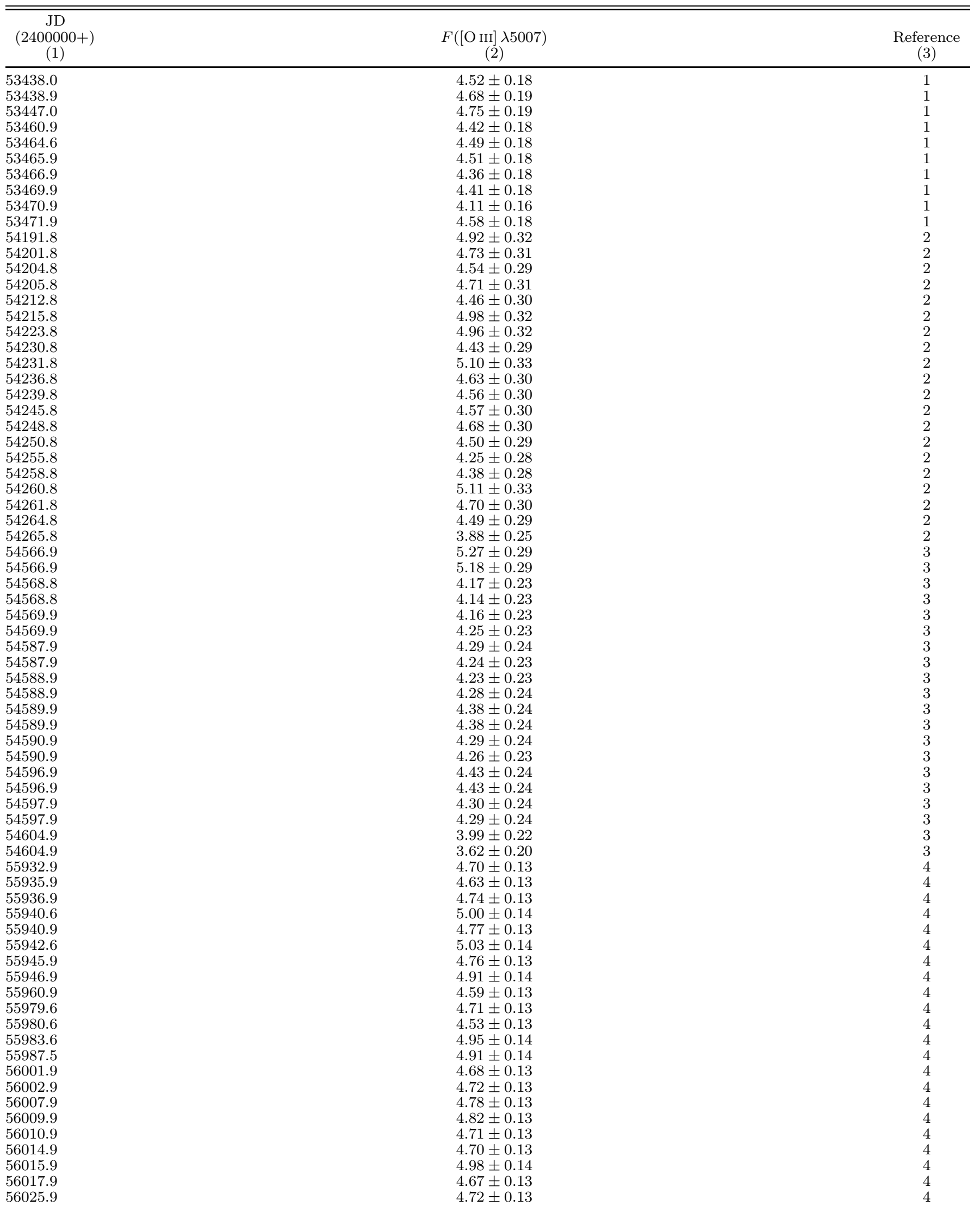

Note. - Column (1) gives the Julian Date of observation. Column (2) gives the observed frame [O III] $\lambda 5007$ emission line flux and its estimated uncertainty in units of $10^{-13} \mathrm{ergs} \mathrm{s}^{-1} \mathrm{~cm}^{-2}$. Column (3) gives the reference for each spectrum. References: (1) AGN05 (Bentz et al. 2007); (2) AGN07 (Dennev et al. 2010); (3) LAMP08 (Bentz et al. 2009b); (4) AGN12 (De Rosa et al. 2013). 
TABLE 3

Adopted Mean [O iit] $\lambda 5007$ Fluxes and Correction Factors

\begin{tabular}{|c|c|c|c|c|}
\hline $\begin{array}{l}\text { Data Set } \\
\text { (1) }\end{array}$ & $\begin{array}{l}\text { Ref. } \\
(2)\end{array}$ & $\begin{array}{c}\text { JD Range } \\
(2400000+) \\
(3)\end{array}$ & $\langle F([\mathrm{O}$ III $] \lambda 5007)\rangle$ & $\begin{array}{c}\text { Flux } \\
\text { Correction } \\
\text { Factor } \\
(5)\end{array}$ \\
\hline AGN Watch, Yr 1 (1988-89) & 1 & $47509-47809$ & $5.586 \pm 0.278$ & 1.001 \\
\hline AGN Watch, Yr 2 (1989-90) & 2 & $47861-48179$ & $5.484 \pm 0.239$ & 0.983 \\
\hline AGN Watch, Yr 3 (1990-91) & 3 & $48225-48534$ & $5.396 \pm 0.165$ & 0.967 \\
\hline AGN Watch, Yr 4 (1991-92) & 3 & $48623-48898$ & $5.519 \pm 0.280$ & 0.989 \\
\hline AGN Watch, Yr 5 (1992-93) & 4 & $48954-49256$ & $5.620 \pm 0.172$ & 1.007 \\
\hline AGN Watch, Yr 6 (1993-94) & 5 & $49309-49637$ & $5.355 \pm 0.517$ & 0.960 \\
\hline AGN Watch, Yr 7 (1994-95) & 5 & $49679-50008$ & $5.386 \pm 0.125$ & 0.965 \\
\hline AGN Watch, Yr 8 (1995-96) & 6 & $50044-50373$ & $5.163 \pm 0.346$ & 0.925 \\
\hline AGN Watch, Yr 9 (1996-97) & 6 & $50435-50729$ & $5.162 \pm 0.220$ & 0.925 \\
\hline AGN Watch, Yr 10 (1997-98) & 6 & $50775-51085$ & $5.013 \pm 0.350$ & 0.898 \\
\hline AGN Watch, Yr 11 (1998-99) & 6 & $51142-51456$ & $5.022 \pm 0.263$ & 0.900 \\
\hline AGN Watch, Yr 12 (1999-2000) & 6 & $51517-51791$ & & 0.882 \\
\hline AGN Watch, Yr 13 (2000-01) & 6 & $51879-52265$ & $4.820 \pm 0.212$ & 0.864 \\
\hline AGN05 (Bentz et al. 2007) & 6 & $53431-53472$ & $4.485 \pm 0.178$ & 0.804 \\
\hline AGN07 (Denney et al. 2010) & 6 & $54180-54333$ & $4.629 \pm 0.298$ & 0.830 \\
\hline LAMP08 (Bentz et al. 2009b) & 6 & $54509-54617$ & $4.330 \pm 0.353$ & 0.776 \\
\hline AGN12 (De Rosa et al. 2013) & 6 & $55932-56048$ & $4.772 \pm 0.136$ & 0.855 \\
\hline
\end{tabular}

Noте. - The monitoring campaign name and reference are given in columns (1) and (2) and the range of Julian Dates is given in columnn (3). The average and standard deviation $[\mathrm{O}$ III $] \lambda 5007$ emission-line fluxes are given in column (4) in units of $10^{-13} \mathrm{ergs} \mathrm{s}^{-1} \mathrm{~cm}^{-2}$. Column (5) is the ratio of the flux given in column (4) to the previously adopted absolute flux of $F([\mathrm{O}$ III $] \lambda 5007)=5.58 \times 10^{-13} \mathrm{ergs} \mathrm{s}^{-1} \mathrm{~cm}^{-2}$. References: (1) Peterson et al. (1991); (2) Peterson et al. (1992); (3) Peterson et al. (1994); (4) Korista et al. (1995); (5) Peterson et al. (1999); (6) This work.

TABLE 4

Host Galaxy Contributions

\begin{tabular}{lcc}
\hline \hline \multicolumn{1}{c}{$\begin{array}{c}\text { Data Set } \\
(1)\end{array}$} & $\begin{array}{c}\text { Aperture Geometry }(\operatorname{arcsec}) \\
(2)\end{array}$ & $\begin{array}{c}F_{\text {gal }}[5100 \AA(1+z)] \\
(3)\end{array}$ \\
\hline AGN Watch, Years 1-13 & $5 \times 7.5$ & $3.75 \pm 0.38$ \\
AGN05 & $5 \times 12.75$ & $4.34 \pm 0.43$ \\
AGN07 & $5 \times 12.0$ & $4.27 \pm 0.43$ \\
LAMP08 & $4 \times 9.4$ & $3.54 \pm 0.35$ \\
AGN12 & $5 \times 15.0$ & $4.45 \pm 0.44$
\end{tabular}

Note. - Column (1) identifies the individual monitoring campaigns. Column (2) gives the nominal spectrograph entrance aperture (projected slit width and extraction window) used in each campaign. Column (3) gives the adopted $5100 \AA$ observed frame hostgalaxy flux through that aperture, based on the nucleus-free model of the NGC 5548 host galaxy from (Bentz et al. (2013), in units of $10^{-15} \operatorname{ergs~s}^{-1} \mathrm{~cm}^{-2} \AA^{-1}$. 
TABLE 5

Revised Continuum Light Curve

\begin{tabular}{|c|c|}
\hline $\begin{array}{c}\text { Julian Date } \\
\text { (1) }\end{array}$ & $\begin{array}{c}F_{\lambda}(5100 \AA[1+z]) \\
(2)\end{array}$ \\
\hline $\begin{array}{l}47509.000 \\
47512.000 \\
47517.000 \\
47524.000 \\
47525.000 \\
47528.000 \\
47533.000 \\
47534.000 \\
47535.000 \\
47539.000\end{array}$ & $\begin{array}{l}5.239 \pm 0.360 \\
5.990 \pm 0.731 \\
5.970 \pm 0.390 \\
6.050 \pm 0.390 \\
6.370 \pm 0.400 \\
6.821 \pm 0.420 \\
6.400 \pm 0.410 \\
6.811 \pm 0.420 \\
6.420 \pm 0.410 \\
7.001 \pm 0.380\end{array}$ \\
\hline
\end{tabular}

Note. - A complete version of Table 5 is available in machine-readable form. Column (1) is the Julian Date -2400000. Column (2) gives the observed-frame AGN flux at rest wavelength $5100 \AA$, corrected for the host galaxy contribution, in units of $10^{-15} \mathrm{ergs} \mathrm{s}^{-1} \mathrm{~cm}^{-2} \AA^{-1}$.

TABLE 6

Revised H $\beta$ Emission-Line Light Curve

\begin{tabular}{cr}
\hline \hline $\begin{array}{l}\text { Julian Date } \\
(1)\end{array}$ & $F(\mathrm{H} \beta)$ \\
\hline 47509.000 & $(2)$ \\
47512.000 & $6.924 \pm 0.260$ \\
47517.000 & $7.634 \pm 0.450$ \\
47524.000 & $7.424 \pm 0.280$ \\
47525.000 & $8.135 \pm 0.310$ \\
47528.000 & $8.025 \pm 0.300$ \\
47530.000 & $7.704 \pm 0.290$ \\
47533.000 & $7.484 \pm 0.280$ \\
47534.000 & $7.915 \pm 0.300$ \\
47535.000 & $7.654 \pm 0.290$ \\
\hline
\end{tabular}

Note. - A complete version of Table 6 is available in machine-readable form. Column (1) is the Julian Date -2400000. Column (2) gives the observed-frame $\mathrm{H} \beta$ emission-line flux, corrected for the $\mathrm{H} \beta$ narrow component, in units of $10^{-13} \mathrm{ergs} \mathrm{s}^{-1} \mathrm{~cm}^{-2}$. 


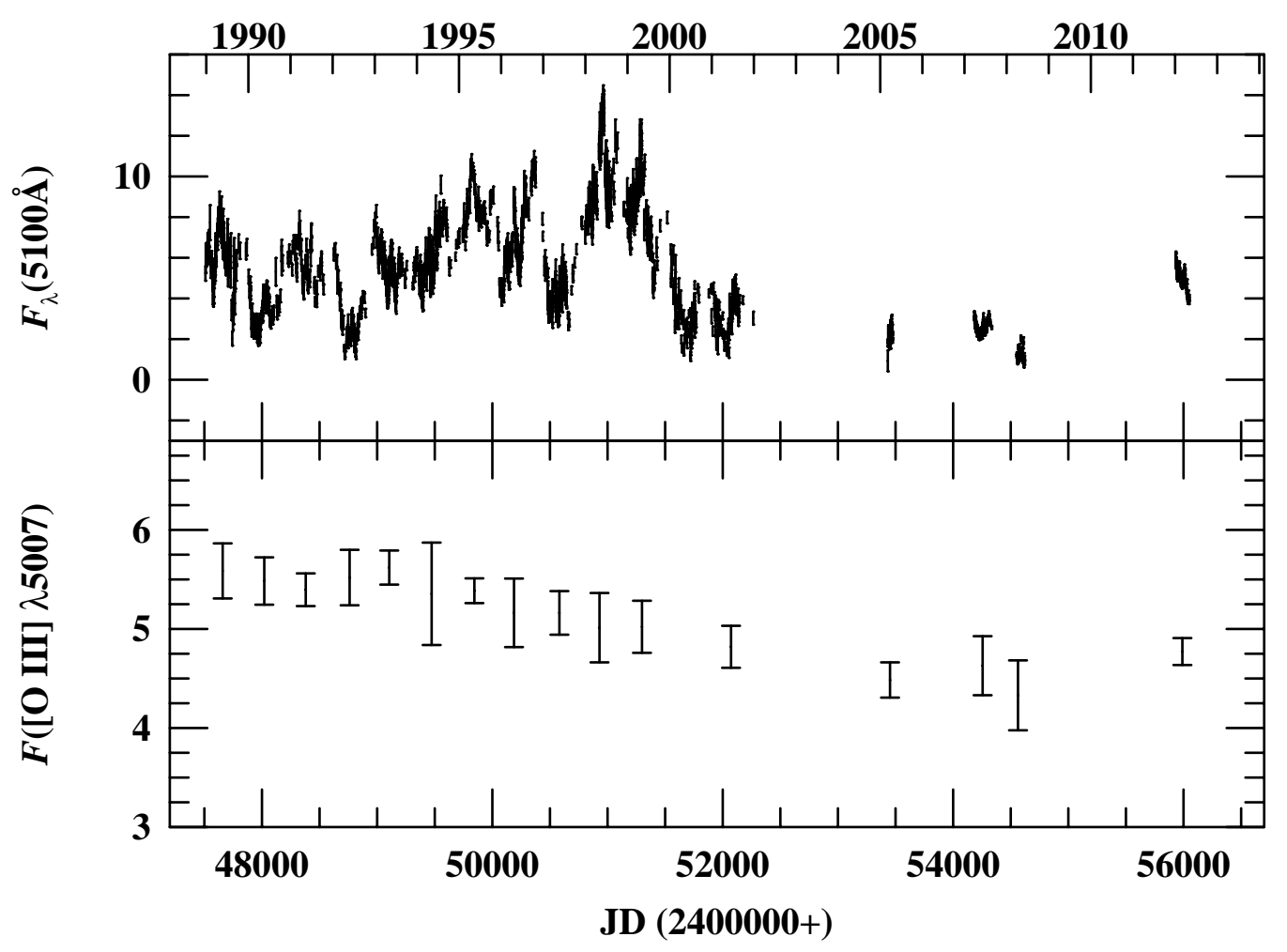

FIG. 1.- Top panel: Observed frame $5100 \AA$ AGN continuum flux as a function of time, calibrated to a constant value of the [O III] $\lambda 5007$ flux with a host-galaxy contribution (Table 4) removed, in units of $10^{-15} \mathrm{ergs}^{-1} \mathrm{~cm}^{-2} \AA^{-1}$. Original sources of data are cited in the notes to Table 3. Bottom panel: Observed frame time-averaged [O III] $\lambda 5007$ fluxes measured from spectra taken on nights that observers recorded night-sky conditions to be "clear" or "photometric," in units of $10^{-13} \mathrm{ergs}^{-1} \mathrm{~cm}^{-2}$, as given in Table 3 . 


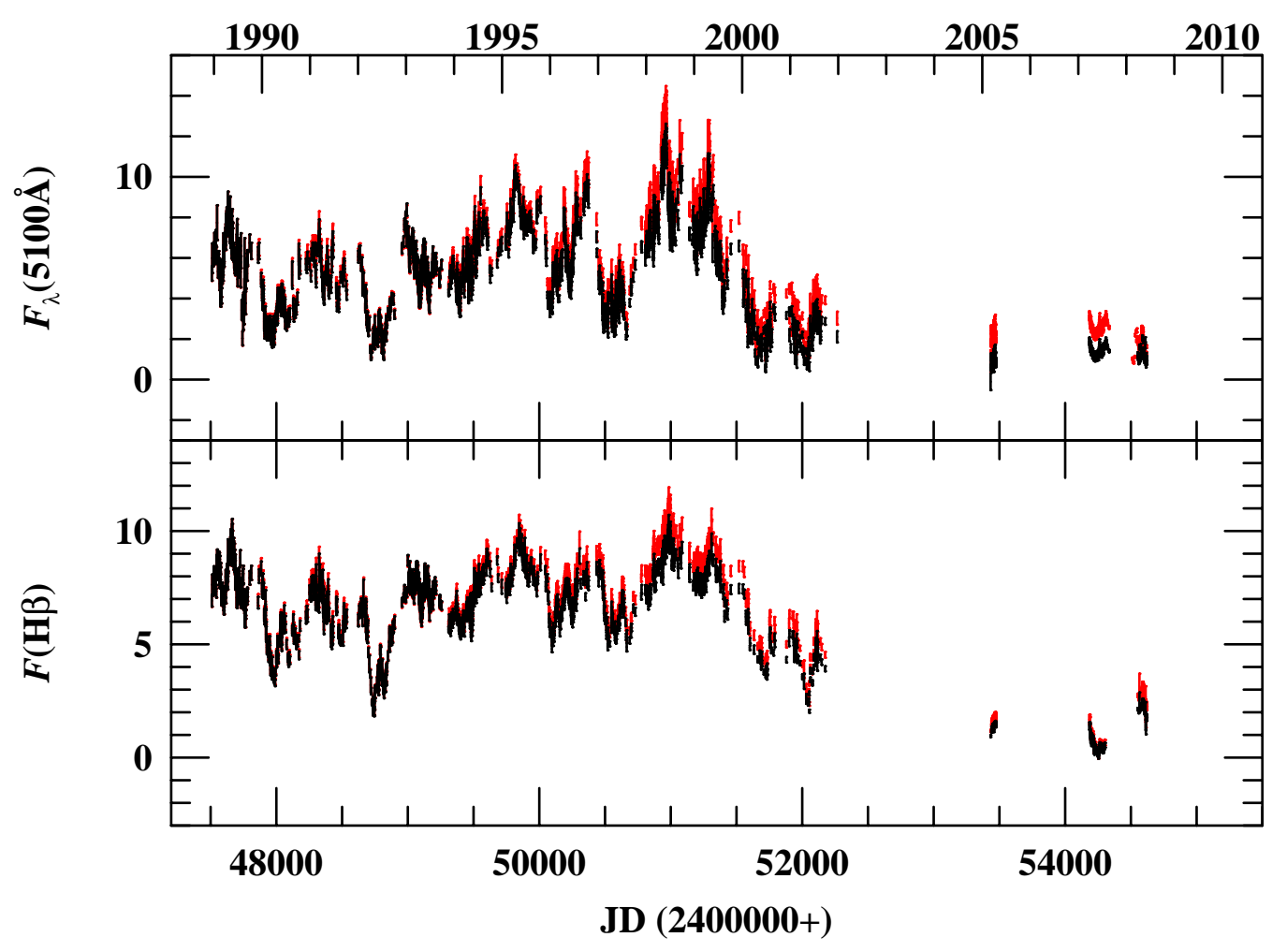

FIG. 2. - Recalibrated observed-frame AGN continuum (top panel, in units of $10^{-15} \mathrm{ergs}^{-1} \mathrm{~cm}^{-2} \AA^{-1}$ ) and broad H $\beta$ emission-line (bottom panel, in units of $10^{-13} \mathrm{ergs}^{-1} \mathrm{~cm}^{-2}$ ) light curves for NGC 5548. The lower axis shows the Julian Date and the upper axis shows calendar year. The light curves are flux calibrated to the [O III $\lambda 5007$ fluxes given in Table 3 and shown in Figure 1 . The continuum has been corrected for the host galaxy contribution as given in Table 4, and a narrow $\mathrm{H} \beta$ contribution has been subtracted from the broad $\mathrm{H} \beta$ light curve assuming a narrow line flux $11 \%$ that of $[\mathrm{O} I I] \lambda 5007$. The original light curves are plotted in red, and the recalibrated light curves are plotted over them in black. 


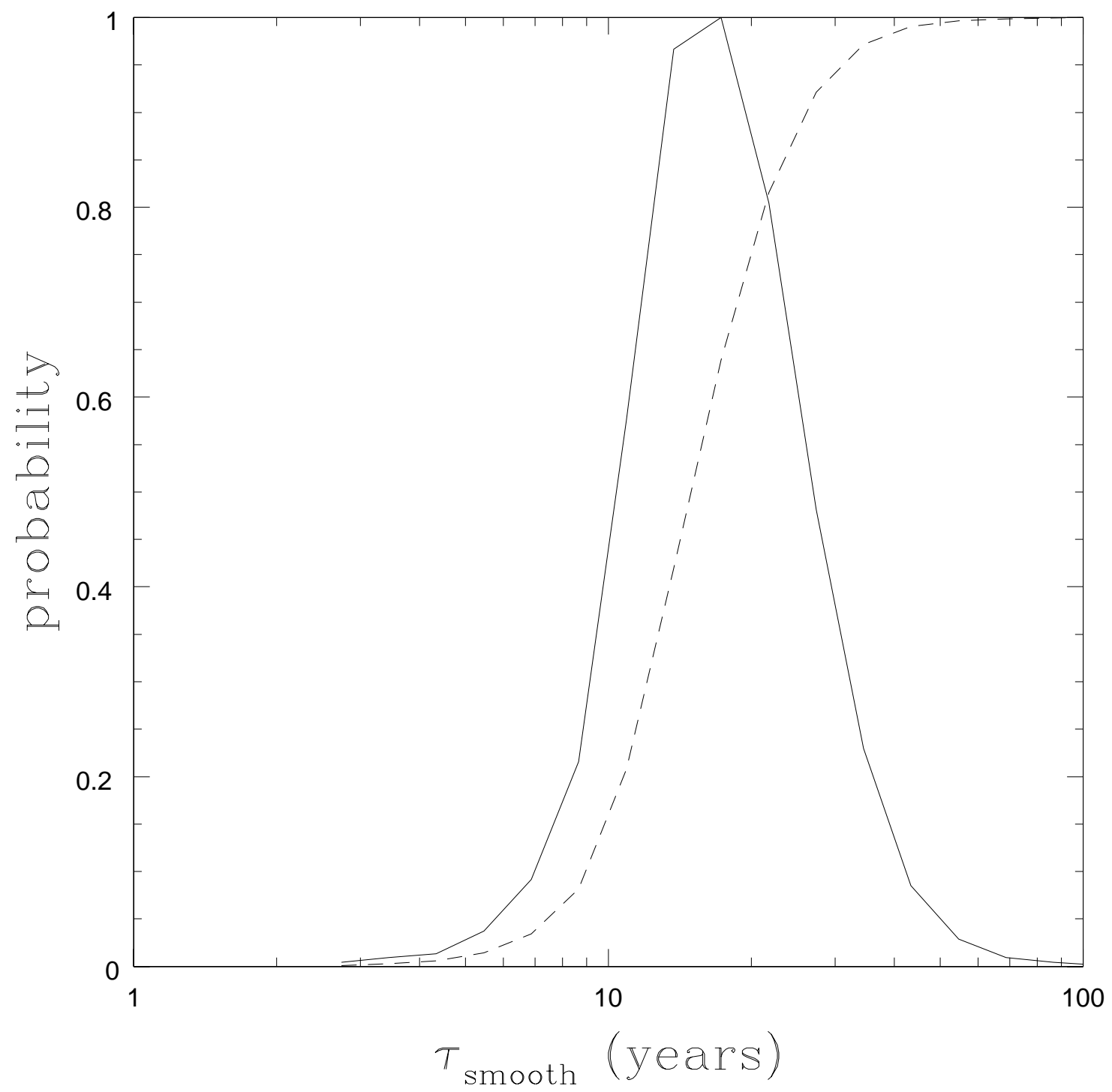

FIG. 3.- Differential (solid) and integral (dashed) probability distributions for the smoothing time scale $\tau_{\text {smooth }}$ required to match the continuum variability to the narrow line variability. The differential probability distribution is normalized to have a peak of unity. This does not include the correction for the seasonal averaging of the [O III] fluxes. 


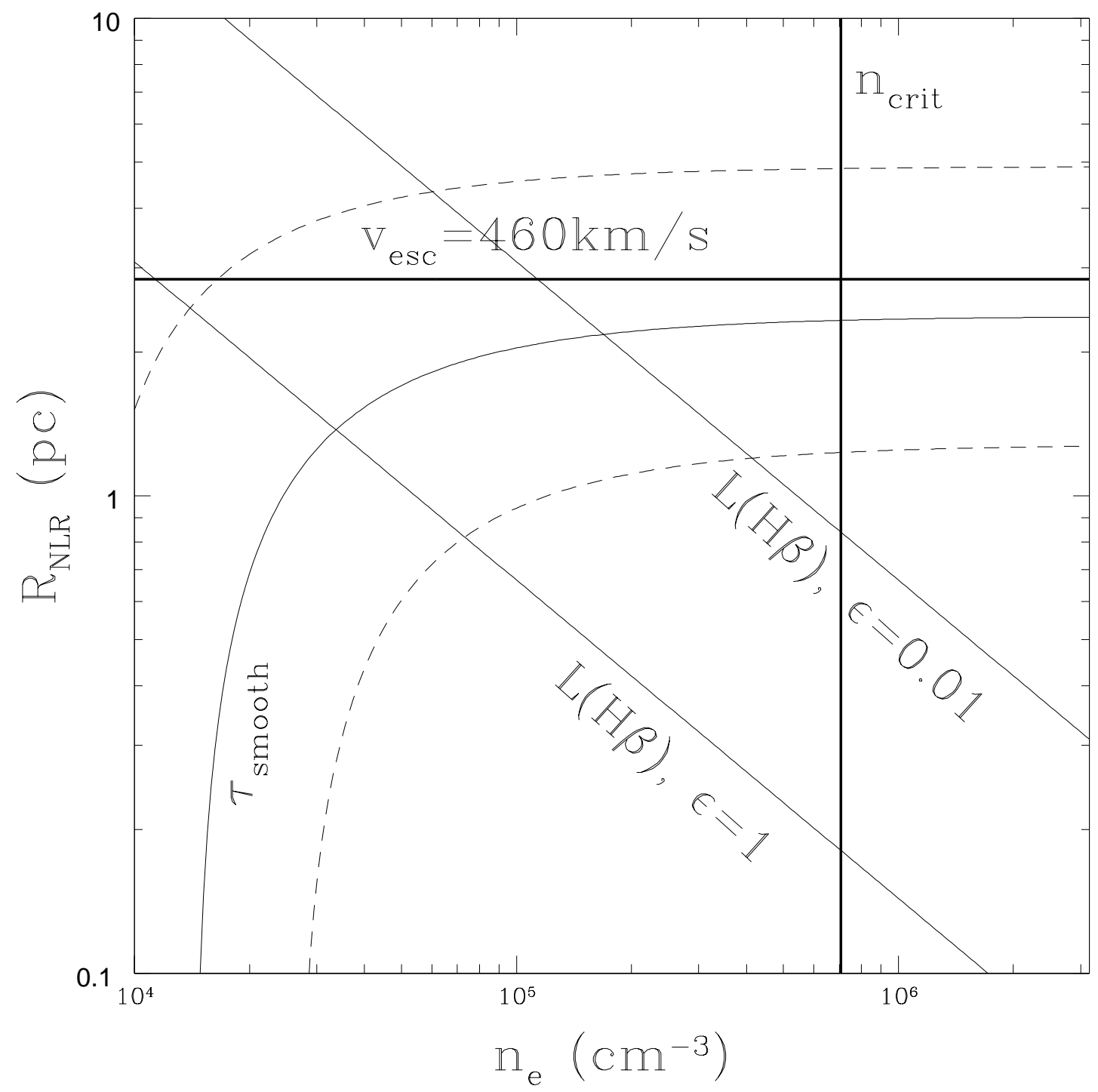

FIG. 4.- Constraints on the narrow line region density $n_{e}$ and size $R_{\mathrm{NLR}}$. The curves labeled $\tau_{\mathrm{smooth}}$ show the median (solid) and $90 \%$ confidence limits (dashed) from the observed narrow-line variability. The lines labeled $L(\mathrm{H} \beta)$ show the conditions needed to produce the observed $\mathrm{H} \beta$ luminosity for a filling factor of either $\epsilon=1$ or 0.01 . The heavy vertical line indicates the critical density for [O III] emission. The heavy horizontal line indicates the radius where the escape velocity is $v_{\text {esc }}=460 \mathrm{~km} \mathrm{~s}^{-1}$ for $M_{\mathrm{BH}}=7 \times 10^{7} M_{\odot}$. 


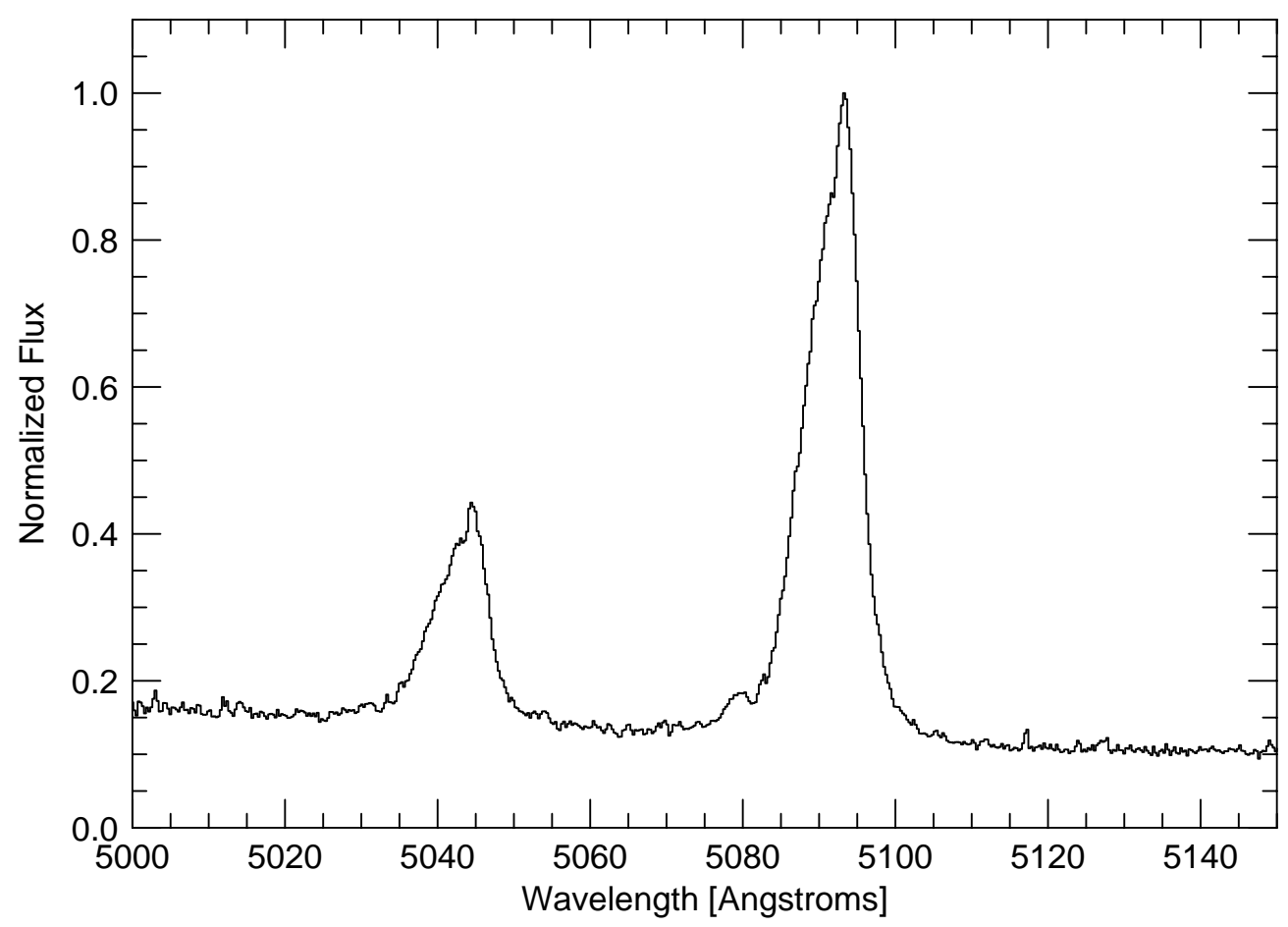

FIG. 5.- HST STIS spectrum of the [O III] $\lambda \lambda 4959,5007$ lines in NGC 5548. 